\title{
The crystal structure of p-type transparent conductive oxide $\mathrm{CuBO}_{2}$
}

\author{
Tiago F.T. Cerqueira and Rafael Sarmiento-Pérez, Institut Lumière Matière, UMR5306 Université Lyon 1-CNRS, Université de Lyon, F-69622 \\ Villeurbanne Cedex, France \\ Fabio Trani, Institute of Theoretical Physics, École Polytechnique Fédérale de Lausanne (EPFL), CH-1015 Lausanne, Switzerland \\ Maximilian Amsler and Stefan Goedecker, Department of Physics, Universität Basel, Klingelbergstr. 82, 4056 Basel, Switzerland \\ Miguel A.L. Marques and Silvana Botti, European Theoretical Spectroscopy Facility \\ Address all correspondence to Silvana Botti at silvana.botti@univ-Iyon1.fr
}

(Received 28 March 2013; accepted 12 June 2013)

\begin{abstract}
We employed ab initio global structural prediction algorithms to obtain the ground-state structure of $\mathrm{CuBO}_{2}$ This is a very promising $\mathrm{p}$-type transparent conductive oxide that was synthesized recently, and thought to belong to the delafossite family. We proved that the true ground state is certainly not the delafossite structure, and that the most promising candidate is a low symmetry monoclinic phase. This is still a layered structure, but with boron and copper having a different coordination with respect to the delafossite phase.
\end{abstract}

By reporting in 1997, a transparent semiconducting material (the delafossite $\mathrm{CuAlO}_{2}$ ) with "reasonable" p-type conductivity, Kawazoe [1] and his co-workers opened a new era of "invisible electronics" [2] built on active devices such as diodes. This led to the opening of a variety of fields, including transparent electronics and opto-electronics, organic lightemitting diodes, integrated electro-optical (waveguide) sensors, novel solar cells, and functional smart windows.

The major ingredient missing for large-scale development of such technologies is a p-type semiconductor with a large gap, high conductivities and mobilities, together with controlled transparencies that can be manufactured industrially. The most promising materials are still probably the delafossites, such as the original p-type transparent conductive oxide (TCO), $\mathrm{CuAlO}_{2}[1]$.

From all the $\mathrm{Cu}$-based delafossites, $\mathrm{CuBO}_{2}$ is particularly interesting as it possesses large band gaps (direct and indirect) and higher intrinsic conductivity than any other $\mathrm{Cu}$-based TCO [3]. Furthermore, it has a structure symmetry compatible with $\alpha-\mathrm{Al}_{2} \mathrm{O}_{3}$ sapphire and $\mathrm{ZnO}$, the latter being already widely used as n-type TCO. This last point is important for the design of a $\mathrm{p}-\mathrm{n}$ junction, the initial brick of any active electrical component.

$\mathrm{CuBO}_{2}$ thin films were for the first time synthesized and characterized in 2007 by Snure and Tiwari [3]. However, a theoretical work by Scanlon et al. [4] questioned the validity of the experimental lattice parameters, as they were in disagreement by more than $10 \%$ with the theoretical values obtained using either the local density approximation or the HeyidScuseria-Ernzerhof (HSE06) [5] functional. This result was quite unexpected, as density functional theory (DFT) calculations usually (and, in particular, for the other delafossite structures) give good estimations of the lattice parameters, within a couple of percent. Moreover, the direct band gap calculated with accurate many-body methods for the $\mathrm{CuBO}_{2}$ delafossite structure relaxed within the local density approximation yielded $3.52 \mathrm{eV}$ [6], much smaller than the reported experimental optical gap of $4.5 \mathrm{eV}$ [3]. On the other hand, the same technique yielded, for other delafossites $\left(\mathrm{CuAlO}_{2}, \mathrm{CuGaO}_{2}\right.$, and $\mathrm{CuInO}_{2}$ ), direct band gaps consistently larger than experiment [6] (with the difference being due to excitonic effects and lattice contribution to the screening [26]). These facts were very surprising, and led to identifying $\mathrm{CuBO}_{2}$ as an atypical compound within the class of $\mathrm{Cu}$ delafossites.

To shed more light on this controversial issue, we performed global structural prediction calculations for the $\mathrm{CuBO}_{2}$ system to determine the ground-state structure of this material. We employed the minima hopping method (MHM) [8,9], an efficient crystal structure prediction algorithm designed to find the low-energy structures of a system, given solely its chemical composition. The energy surface is explored by performing consecutive short molecular dynamics escape steps followed by local geometry relaxations, taking into account both atomic and cell variables. The initial velocities for the molecular dynamics trajectories are chosen approximately along softmode directions, thus allowing efficient escapes from local minima and aiming toward low-energy structures. The predictive power of this approach has been demonstrated in a wide range of applications [10-12]. We used cells containing 1, 2, and 3 formula units (up to 12 atoms), and forces and energies were obtained using DFT as implemented in the code VASP [13] with the Perdew-Burke-Ernzerhof approximation (PBE) [14] to the exchange-correlation functional. 
We note that there are two possible stacking configurations available for the delafossite structure: hexagonal (space group $P 63 / m m c$ ) and rhombohedral (space group $R \overline{3} m$ ) [15], with very similar energies, density of states, and bond lengths [16]. Both structures did indeed appear in our simulations; however, they were not, by far, the lowest energy phases. In our extensive structural search, we could not find any lowenergy local minimum with lattice constants similar to the ones given by Snure and Tiwari [3]. The most stable structure turned out to be a low-symmetry atomic arrangement, that ISOTROPY [17] classified as belonging to space group $C c$ with $a=3.63 \AA, b=12.45 \AA, c=4.42 \AA, \alpha=\gamma=90^{\circ}$, and $\beta=$ $86.78^{\circ}$, with the $\mathrm{Cu}$ atoms at the $4 a$ Wyckoff position $(-0.0055,0.2465,0.0439), \mathrm{B}$ at the $4 a$ Wyckoff position $(-0.3632,0.4496,0.1981)$, and two non-equivalent $\mathrm{O}$ at the $4 a$ Wyckoff positions $(0.2555,0.1490,-0.2105)$ and (0.0791, 0.0349, 0.3937). This crystal structure, which can be seen in the lower panel of Fig. 1, has planar $\mathrm{Cu}$ layers intercalated by linear $\mathrm{BO}_{2}$ networks where $\mathrm{B}$ is threefold coordinated.

Other interesting high-symmetry phases found during our MHM runs were a body centered tetragonal phase (space group $I \overline{4} m 2$ ) and an orthorhombic phase (space group $C m c 2_{1}$ ) (see Fig. 1). Like the delafossite and the $C c$ structure, they have a layered geometry, consisting in alternating planar layers of $\mathrm{Cu}$ and layers of $\mathrm{BO}_{2}$. However, for the tetragonal structure $\mathrm{Cu}$ atoms form a square planar lattice (and not an hexagonal as in the delafossites), while B atoms are also fourfold coordinated (and not sixfold as in the delafossite). This tetragonal phase has $a=2.54 \AA$ and $c=10.82 \AA$, with $\mathrm{Cu}$ at the Wyckoff position (a), B at the Wyckoff position (d), and

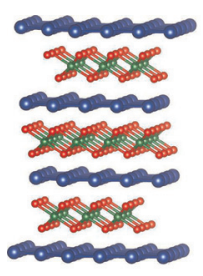

$R \overline{3} m$

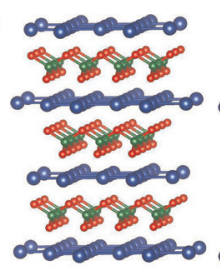

$I \overline{4} m 2$

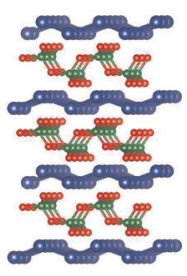

$C m c 2_{1}$

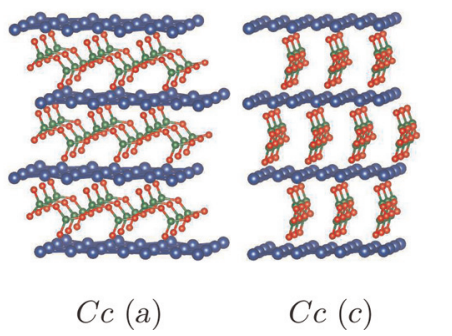

Figure 1. (Color online) Different crystal structures of $\mathrm{CuBO}_{2}$ studies in this work. Top panel: delafossite $(R \overline{3} m)$, tetragonal $(I \overline{4} m 2)$, orthorhombic $\left(C m 2_{1}\right)$. Bottom panel: two views of the monoclinic $(C c)$ space group along the $a$ and $c$ crystallographic directions. the two $\mathrm{O}$ atoms at Wyckoff positions (e) with $z=0.175$. On the other hand, the orthorhombic phase is composed of corrugated hexagonal $\mathrm{Cu}$ layers, separated by $\mathrm{BO}_{2}$ layers where $\mathrm{B}$ is fourfold coordinated. The orthorhombic phase has $a=$ $2.57 \AA, b=11.67 \AA$, and $c=4.51 \AA$, with $\mathrm{Cu}$ at the $4 a$ Wyckoff position $(-0.2118,-0.4770), \mathrm{B}$ at the $4 a$ Wyckoff position $(-0.0571,0.0860)$, and two non-equivalent $\mathrm{O}$ atoms att he $4 a$ Wyckoff positions $(-0.3770,-0.4942)$ and (0.0477, -0.0862).

The $C c$ structure lies almost $0.6 \mathrm{eV}$ per atom lower in energy than the $R \overline{3} m$ delafossite phase, while the $C m c 2_{1}$ and $I \overline{4} m 2$ phases are almost degenerate at around $-0.5 \mathrm{eV}$ per atom. Furthermore, many other phases (mostly of very low symmetry) were found in an energy range between the ground state and the delafossite structure. Finally, we also performed, as a cross-check, minima hopping runs for $\mathrm{CuAlO}_{2}, \mathrm{CuGaO}_{2}$, and $\mathrm{CuInO}_{2}$. All these runs yielded invariably the delafossite structure as the ground state of these systems. Note that, in these cases, the delafossite structure was reached within very few steps (usually two or three) during the minima hopping runs, and temperatures in excess of $10^{4} \mathrm{~K}$ were required to exit these valleys. Finally, we note that the lower symmetry $C c$ and $C m c 2_{1}$ structures are not stable for $\mathrm{CuAlO}_{2}, \mathrm{CuGaO}_{2}$, and $\mathrm{CuInO}_{2}$.

Although an energy difference of $0.6 \mathrm{eV}$ is considerably larger than the typical error incurred in PBE calculations, we performed calculations (using the PBE geometry) at higher levels of theory and for the other delafossites to confirm our findings. We used the HSE06 screened hybrid [5], the PBE0 hybrid [18], and GGA + U in the framework of Ref. 19 (we set $U-J=5 \mathrm{eV}$ for the $d$ states of $\mathrm{Cu}$ ). Our findings are summarized in Table 1. Results for the $P 63 / m m c$ phase were virtually identical (to a few $\mathrm{meV}$ ) to the $R \overline{3} \mathrm{~m}$ structure and are therefore not shown in the table.

Remarkably, the four methods (PBE, GGA + U, HSE06, and PBE0) yield essentially the same energy differences for all structures and compounds studied $\left(\mathrm{CuBO}_{2}, \mathrm{CuAlO}_{2}\right.$, $\mathrm{CuGaO}_{2}$, and $\mathrm{CuInO}_{2}$ ). Furthermore, and confirming our minima hopping simulations, the $C c$ structure is only the ground state for $\mathrm{CuBO}_{2}$. Finally, we see that the $R \overline{3} m$ phase is stabilized with respect to the $I \overline{4} m 2$ structure with increasing size of the trivalent metal (i.e., descending the IIIA column). This means that probably only B crystallizes in this tetragonal structure as it is the only trivalent metal smaller than Al, making this phase quite unique.

A possible explanation for destabilization of the delafossite phase of $\mathrm{CuBO}_{2}$ can be found by analyzing the oxidation state of the metal. In fact, in the delafossite structure, the metal atom is in the oxidation state +3 . However, on performing a Bader analysis of delafossite $\mathrm{CuBO}_{2}$ one finds a Bader charge for $\mathrm{B}$ of +2.15 , to compare with +2.54 for $\mathrm{Al}$ in $\mathrm{CuAlO}_{2}$. Note that this charge increases to +2.30 in $I \overline{4} m 2 \mathrm{CuBO}_{2}$ and +2.31 in $C c$.

All the considered phases of $\mathrm{CuBO}_{2}$ turned out to have indirect fundamental band gaps, and all band gaps are large 
Table 1. Energy per atom (in meV) of different structures of $\mathrm{CuXO}_{2}$ studied in this work using different theoretical methods. The zero is the energy of the delafossite $R \overline{3} m$ structure

\begin{tabular}{|c|c|c|c|c|c|c|}
\hline$x$ & $\begin{array}{l}B \\
C c\end{array}$ & $\begin{array}{c}\mathrm{B} \\
\mathrm{Cmc} 2_{1}\end{array}$ & $\begin{array}{c}\text { B } \\
\bar{I} m_{2}\end{array}$ & $\begin{array}{c}\mathrm{Al} \\
\overline{I \overline{4}} \mathrm{~m} 2\end{array}$ & $\begin{array}{c}\mathrm{Ga} \\
\overline{1} \overline{4} \mathrm{~m}^{2}\end{array}$ & $\begin{array}{c}\text { In } \\
\mid \overline{4} m 2\end{array}$ \\
\hline PBE & -569 & -483 & -482 & 123 & 138 & 248 \\
\hline$G G A+U$ & -598 & -508 & -491 & 113 & 128 & 236 \\
\hline HSE06 & -660 & -565 & -555 & 124 & 135 & 262 \\
\hline PBE0 & -659 & -566 & -546 & 131 & 143 & 289 \\
\hline
\end{tabular}

enough to assure transparency. The $I \overline{4} m 2$ phase has a calculated indirect gap of $4.0 \mathrm{eV}$, whereas the monoclinic $C c$ and orthorombic $C m c 2_{1}$ phases have very similar indirect band gaps of 4.0 and $4.2 \mathrm{eV}$, respectively. These band gaps are larger than the corresponding band gap for the delafossite structure $(3.7 \mathrm{eV})$. Note that we chose PBE0 values as we expect them to give the best estimate, in analogy with previous calculations for other members of the delafossite family [6,7]. The experimental indirect band gap reported in Ref. 3 is $2.2 \mathrm{eV}$. Once again, as it happens for $\mathrm{CuAlO}_{2}, \mathrm{CuGaO}_{2}$, and $\mathrm{CuInO}_{2}$, the calculated indirect band gap is much larger than the experimental gap. This finding supports the recent view that the low-energy absorption bands of this class of TCOs are probably due to defect states in the gap [6, 20-22].

The calculated direct band gaps within PBE0 are $4.4 \mathrm{eV}$ for the $I \overline{4} m 2$ crystal, $4.2 \mathrm{eV}$ for the $C c$ phase, $4.2 \mathrm{eV}$ for the $C m c 2_{1}$ phase, and $4.7 \mathrm{eV}$ for the delafossite. These values are close to the measured optical direct gap of $4.5 \mathrm{eV}$. However, one should not for get that the fundamental band-edge transitions can be forbidden by selection rules in highly symmetric structures, as it happens in delafossites [4, 23], moving to higher energy the measured absorption edge. For an accurate quantitative comparison of theory and experiments, the excitonic binding energy and renormalization of the gap due to lattice polarization should also be included [26].

The top of the valence band turns out to be of mixed $\mathrm{Cu} d$ with $\mathrm{O} d$ character while the bottom of the conduction is a mixture of $\mathrm{Cu} s, p$, and $d$ states with mainly $\mathrm{O} p$ (and some $\mathrm{O} s$ ) states, and a significant amount of $\mathrm{B} p$. This is similar to the case of the delafossite structure [6]. Moreover, we observed that the projected densities of states of different phases do not show qualitatively different features.

We remark that the main reason as to why it is so difficult to develop high mobility p-type TCOs is the localized $\mathrm{O} p$ nature of the valence band in most oxides, yielding large hole effective masses. In the delafossite structure, and also in the other lowest-energy structures that we have identified, strong hybridization of the $\mathrm{O} p$ states with $\mathrm{Cu} d$ states leads to a dispersive top valence band with a lower effective mass than in other oxides.

The average in-plane hole effective mass of the $C c$ phase is 1.0, to be compared, e.g., with 2.6 for $\mathrm{CuAlO}_{2}$ [24]. On the other hand, the perpendicular mass is 5.0, an order of magnitude smaller than 58.4 for $\mathrm{CuAlO}_{2}$ [24]. This indicates that $\mathrm{CuBO}_{2}$, even if it does not crystallize in the delafossite phase, has the potential to be an excellent p-type TCO.

Another essential requirement for p-type conductivity is the possibility to dope with acceptor defects, the most common hole-killer being $\mathrm{O}$ vacancies. The formation energy of the neutral $\mathrm{O}$ vacancy is around $5 \mathrm{eV}$ in the $C c$ phase, very much in line with the other delafossites. We observed that $\mathrm{Cu}$ vacancies have a large negative formation energy in the delafossite phase, a further indication that this structure is not stable.

Finally, in Fig. 2 we present x-ray diffraction results for different structures of $\mathrm{CuBO}_{2}$. These are quite different from each other and should allow for an experimental identification of any of this phases in samples. Unfortunately, the experimental spectra of Ref. 3 and Ref. 25 are either contaminated or do not have enough resolution to allow for an unambiguous identification. We observe that a peak at $2 \theta \simeq 35^{\circ}$ is present in both experiments and the simulated spectra of the new structures reported in this work, whereas it is absent in the simulated $\mathrm{x}$-ray diffraction spectrum of the delafossite $R \overline{3} \mathrm{~m}$ phase.

In conclusion, we performed global structural optimization for the $\mathrm{CuBO}_{2}$ system. Our results indicate that it is extremely

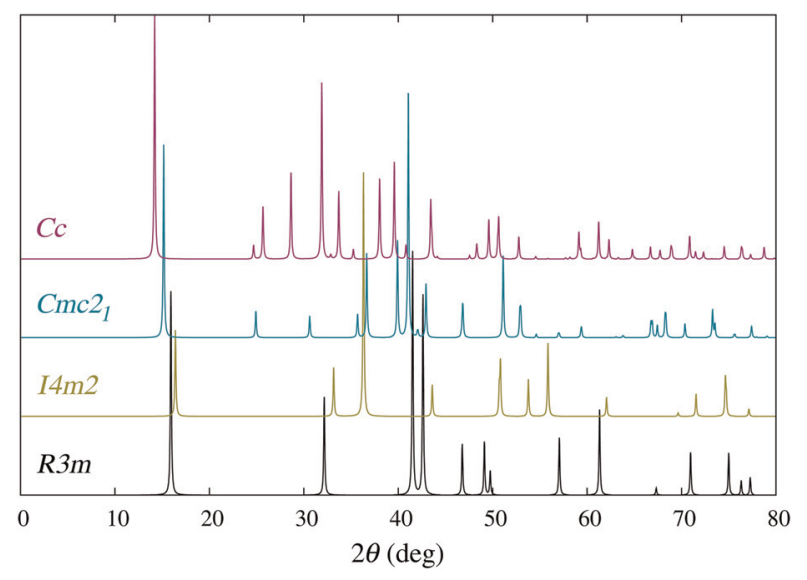

Figure 2. (Color online) Simulated $\mathrm{x}$-ray diffraction spectrum with $\mathrm{Cu} \mathrm{K} \alpha$ radiation $\lambda=1.54178 \AA$. 
unlikely that the experimentally produced phase belongs to the delafossite family. The most likely candidate is instead a low symmetry monoclinic structure, consisting of alternating planar $\mathrm{Cu}$ layers (fourfold coordinated) and $\mathrm{BO}_{2}$ networks (where $\mathrm{B}$ is threefold coordinated). These results call for further experimental work to determine unambiguously the structure of this promising p-type TCO.

\section{ACKNOWLEDGMENTS}

M.A.L.M. and S.B. acknowledge the financial support from the French ANR projects ANR-08-CEXC8-008-01 and ANR-12-BS04-0001-02. Computational resources were provided by GENCI (project x2012096017).

\section{References}

1 H. Kawazoe, M. Yasukawa, H. Hyodo, M. Kurita, H. Yanagi, and $\mathrm{H}$. Hosono: P-type electrical conduction in transparent thin films of CuAl02. Nature, 389, 939 (1997).

2 G. Thomas: Invisible circuits. Nature, 389, 907 (1997).

3 M. Snure and A. Tiwari: A p-type transparent oxide $\mathrm{CuBO}_{2}$. Appl. Phys. Lett., 91, 092123 (2007).

4 D. Scanlon, A. Walsh, and G. Watson: Understanding the p-type conduction properties of the transparent conducting oxide $\mathrm{CuBO}_{2}$ : a density functional theory analysis. Chem. Mater., 21, 4568 (2009).

5 J. Heyd, G. E. Scuseria, and M. Ernzerhof: Erratum: "Hybrid functionals based on a screened coulomb potential". J. Chem. Phys., 124, 219906 (2006).

6 F. Trani, J. Vidal, S. Botti, and M. A. L. Marques: Band structures of delafossite transparent conductive oxides from a self-consistent GW approach. Phys. Rev. B, 82, 085115 (2010).

7 J. Vidal, F. Trani, F. Bruneval, M. A. L. Marques, and S. Botti: Effects of electronic and lattice polarization on the band structure of delafossite transparent conductive oxides. Phys. Rev. Lett., 104, 136401 (2010).

8 S. Goedecker: Minima hopping: an efficient search method for the global minimum of the potential energy surface of complex molecular systems. J. Chem. Phys., 120, 9911 (2004).

9 M. Amsler and S. Goedecker: Crystal structure prediction using the minima hopping method. J. Chem. Phys., 133, 224104 (2010).

10 J. A. Flores-Livas, M. Amsler, T. J. Lenosky, L. Lehtovaara, S. Botti, M. A. Marques, and S. Goedecker: High-pressure structures of disilane and their superconducting properties. Phys. Rev. Lett., 108, 117004 (2012).

11 M. Amsler, J. A. Flores-Livas, T. D. Huan, S. Botti, M. A. Marques, and S. Goedecker: Novel structural motifs in low energy phases of $\mathrm{LiAlH}_{4}$. Phys. Rev. Lett., 108, 205505 (2012).

12 S. Botti, J. A. Flores-Livas, M. Amsler, S. Goedecker, and M. A. L. Marques: Low-energy silicon allotropes with strong absorption in the visible for photovoltaic applications. Phys. Rev. B, 86, 121204 (R) (2012).

13 G. Kresse and J. Furthmüller: Efficient iterative schemes for ab initio total-energy calculations using a plane-wave basis set. Phys. Rev. B, 54, 11169 (1996).

14 J. P. Perdew, K. Burke, and M. Ernzerhof: Generalized gradient approximation made simple. Phys. Rev. Lett., 77, 3865 (1996).

15 B. U. Köhler and M. Jansen: Darstellung und strukturdaten von delafossiten $\mathrm{CuMO}_{2}$ (M = Al, Ga, Sc, Y). Z. Anorg. Allg. Chem., 543, 73 (1986).

16 T. Arnold, D. J. Payne, A. Bourlange, J. P. Hu, R. G. Egdell, L. F. J. Piper, L. Colakerol, A. De Masi, P.-A. Glans, T. Learmonth, K. E. Smith, J. Guo, D. O. Scanlon, A. Walsh, B. J. Morgan, and G. W. Watson: X-ray spectroscopic study of the electronic structure of $\mathrm{CuCrO}_{2}$. Phys. Rev. B, 79, 075102 (2009).

17 H. T. Stokes and D. M. Hatch: J. Appl. Crystallogr., 38, 237 (2005).

18 M. Ernzerhof and G. E. Scuseria: J. Chem. Phys., 110, 5029 (1999).
19 S. L. Dudarev, G. A. Botton, S. Y. Savrasov, C. J. Humphreys, and A. P. Sutton: Electron-energy-loss spectra and the structural stability of nickel oxide: an LSDA + U study. Phys. Rev. B, 57, 1505 (1998).

$20 \mathrm{~J}$. Pellicer-Porres, A. Segura, A. Gilliland, A. Munoz, P. Rodriguez-Hernendez, D. Kim, M. Lee, and T. Kim: On the band gap of $\mathrm{CuAlO}_{2}$ delafossite. Appl. Phys. Lett., 88, 181904 (2006).

21 J. Tate, H. L. Ju, J. C. Moon, A. Zakutayev, A. P. Richard, J. Russell, and D. H. Mclntyre: Origin of p-type conduction in single-crystal CuAlO Phys. Rev. B, 80, 165206 (2009).

22 D. O. Scanlon and G. W. Watson: Conductivity limits in $\mathrm{CuAlO}_{2}$ from screened-hybrid density functional theory. J. Chem. Phys. Lett., 1, 3125 (2010).

23 X. Nie, S. Wei, and S. Zhang: Bipolar doping and band-gap anomalies in delafossite transparent conductive oxides. Phys. Rev. Lett., 88, 066405 (2002).

24 D. 0. Scanlon, K. G. Godinho, B. J. Morgan, and G. W. Watson: Understanding conductivity anomalies in Cu-based delafossite transparent conducting oxides: theoretical insights. J. Chem. Phys., 132, 024707 (2010).

25 S. Santra, N.S. Das, and K.K. Chattopadhyay: Sol-gel synthesis and characterization of wideband gap $\mathrm{p}$-type nanocrystalline $\mathrm{CuBO}_{2}$. Mater Lett., 92, 198 (2013).

26 S. Botti and M.A.L. Marques: Phys. Rev. Lett., 110, 226404 (2013). 